\section{(6) OPEN ACCESS}

\title{
Mitochondrial DNA haplogroups influence the risk of incident knee osteoarthritis in OAI and CHECK cohorts. A meta-analysis and functional study
}

\author{
Mercedes Fernández-Moreno, ${ }^{1}$ Angel Soto-Hermida, ${ }^{1}$ María E Vázquez-Mosquera, ${ }^{1}$ \\ Estefanía Cortés-Pereira, ${ }^{1}$ Sara Relaño, ${ }^{2}$ Tamara Hermida-Gómez, ${ }^{1}$ Sonia Pértega, ${ }^{3}$ \\ Natividad Oreiro-Villar, ${ }^{1}$ Carlos Fernández-López, ${ }^{1}$ Rafael Garesse, ${ }^{4,5}$ \\ Francisco J Blanco, ${ }^{1}$ Ignacio Rego-Pérez ${ }^{1}$
}

\begin{abstract}
Handling editor Tore K Kvien
- Additional material is published online only. To view please visit the journal online (http://dx.doi.org/10.1136/ annrheumdis-2016-210131).

For numbered affiliations see end of article.
\end{abstract}

\section{Correspondence to} Dr Ignacio Rego-Pérez, Rheumatology Service, Genomics Unit, INIBIC-Complejo Hospitalario Universitario A Coruña, A Coruña 15006, Spain: ignacio.rego.perez@sergas.es

FJB and IR-P contributed equally.

Received 28 June 2016 Revised 22 September 2016 Accepted 5 November 2016 Published Online First

5 December 2016

\section{SLinked}

- http://dx.doi.org/10.1136/ annrheumdis-2016-210783

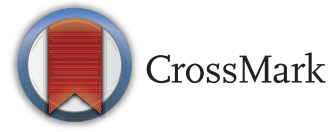

To cite: FernándezMoreno M, Soto-Hermida A, Vázquez-Mosquera ME, et al. Ann Rheum Dis 2017;76:1114-1122.

\section{ABSTRACT}

Objective To evaluate the influence of the mitochondrial DNA (mtDNA) haplogroups in the risk of incident knee osteoarthritis (OA) and to explain the functional consequences of this association to identify potential diagnostic biomarkers and therapeutic targets. Methods Two prospective cohorts contributed participants. The osteoarthritis initiative (OAI) included 2579 subjects of the incidence subcohort, and the cohort hip and cohort knee (CHECK) included 635, both with 8-year follow-up. The analysis included the association of mtDNA haplogroups with the rate of incident knee OA in subjects from both cohorts followed by a subsequent meta-analysis. Transmitochondrial cybrids harbouring haplogroup J or $\mathrm{H}$ were constructed to detect differences between them in relation to physiological features including specific mitochondrial metabolic parameters, reactive oxygen species production, oxidative stress and apoptosis.

Results Compared with $\mathrm{H}$, the haplogroup J associates with decreased risk of incident knee $\mathrm{OA}$ in subjects from OAI (HR=0.680; 95\% Cl 0.470 to $0.968 ; p<0.05)$ and CHECK (HR=0.728; 95\% Cl 0.469 to 0.998; $p<0.05)$. The subsequent meta-analysis including 3214 cases showed that the haplogroup J associates with a lower risk of incident knee $\mathrm{OA}(\mathrm{HR}=0.702 ; 95 \% \mathrm{Cl} 0.541$ to $0.912 ; p=0.008)$. J cybrids show a lower free radical production, higher cell survival under oxidative stress conditions, lower grade of apoptosis as well as lower expression of the mitochondrially related pro-apoptotic gene $\mathrm{BCL} 2$ binding component 3 (BBC3). In addition, $\mathrm{J}$ cybrids also show a lower mitochondrial respiration and glycolysis leading to decreased ATP production. Conclusions The physiological effects of the haplogroup $\mathrm{J}$ are beneficial to have a lower rate of incident knee OA over time. Potential drugs to treat OA could focus on emulating the mitochondrial behaviour of this haplogroup.

\section{INTRODUCTION}

Osteoarthritis (OA) is a chronic progressive disorder involving movable joints characterised by cell stress and extracellular matrix degradation initiated by micro-injury and macro-injury that activates maladaptive repair responses including pro-inflammatory pathways of innate immunity. The disease manifests first as a molecular derangement (abnormal tissue metabolism) followed by anatomical and/or physiological derangements (characterised by cartilage degradation, bone remodelling, osteophyte formation, joint inflammation and loss of normal joint function) that can culminate in illness. ${ }^{1}$

$\mathrm{OA}$ is the most common form of arthritis, occurring in $10 \%-20 \%$ of the population aged over 50 years, and it is estimated that the population with OA will double in the next 30 years. ${ }^{2}$ Thus, the identification of risk factors that accelerate disease progression is critical since these factors could become potential targets for disease modification. ${ }^{3}$ To achieve this objective, the use of wellcharacterised study cohorts to identify prognostic factors that predict the course of OA, as well as to identify markers for the (early) diagnosis and course of joint damage, becomes imperative. In this sense, both osteoarthritis initiative (OAI) and cohort hip and cohort knee (CHECK) stand out.

$\mathrm{OA}$ is a heterogeneous disease in which a combination of modifiable (ie, body mass index (BMI), joint injury) and non-modifiable factors (ie, age, gender, genetics) interact. Thus, the pathogenesis of OA is still unclear; however, it is widely accepted that genetics plays a main role in the prevalence and progression of this disease $;^{4}$ even prediction tools for knee OA based on genetic and clinical information have been developed..$^{5}{ }^{6}$ Besides, during the last years, increasing evidence points to the implication of the mitochondria and the mitochondrial DNA (mtDNA) haplogroups in the pathogenesis of the disease. $^{2} 78$

The mtDNA haplogroups are the result of maternally inherited mutations in the mtDNA acquired throughout the human history and shaped by the climate selection when humans migrated into colder climates. ${ }^{9} 10$ Each of the mtDNA haplogroups harbours specific single nucleotide polymorphisms (SNPs) that influence the behaviour of the mitochondria ${ }^{11}$ and interact with the nuclear genome, ${ }^{12}$ influencing our health today. ${ }^{10}$ Some of these genetic variants have been associated with degenerative disorders, ${ }^{13}$ metabolic alterations ${ }^{14}$ or even increased longevity in humans. ${ }^{15}$

Regarding OA, several studies also reported the association of specific haplogroups with a lower prevalence of the disease, ${ }^{16-19}$ while others did not. ${ }^{20}$ Moreover, recent studies showed significant 
associations of specific haplogroups with a lower rate of radiographic progression and cartilage loss over time in different study cohorts worldwide such as the $\mathrm{OAI}^{21}$ and one Spanish cohort. ${ }^{22}$

In an attempt to ascertain the correlation of mtDNA haplogroups and the rate of incident knee OA, in this work we performed a replication study and meta-analysis of two individual studies involving subjects from CHECK and OAI cohorts. In addition, functional studies using transmitochondrial cybrids were performed to ascertain the cellular mechanisms underlying the association of mtDNA variants with the pathogenesis of the disease.

\section{METHODS}

\section{Incident knee OA study in subjects from the OAI and CHECK cohorts}

Participants

The description of CHECK and OAI cohorts is included in the online supplementary methods section. For this study, we included longitudinal data, in terms of Kellgren and Lawrence (KL) grade, of 2579 participants of the incidence subcohort of the OAI and 635 CHECK participants that met the eligibility criteria for an incident knee OA study monitored at predefined intervals for a period of 8 years.

\section{Incident knee OA criteria}

The incidence of radiographic knee OA was defined on joint level (knees separately) following the appropriate proposed criteria for each of the two cohorts. In OAI subjects, we followed the definition of incident knee OA proposed by Felson et $a l^{23}$ consisted in a new-onset $\mathrm{KL}$ grade $\geq 2$ from baseline to follow-up. In CHECK subjects, we followed the proposed definition of incident knee OA for this cohort ${ }^{24} 25$ consisted in a new-onset KL grade $\geq 1$ from baseline to follow-up in accordance with the very early stage OA in CHECK subjects.

\section{mtDNA haplogroups genotyping}

The mtDNA haplogroups were assigned in 2579 DNA samples belonging to the OAI and 635 from CHECK following a previously described assay ${ }^{16}$ (see online supplementary methods).

\section{Statistical analysis}

All the statistical analyses were performed using IBM-SPSS software V.19 (IBM, Armonk, New York, USA) and R software V.3.1.2 (The R Foundation for Statistical Computing). All comparisons were two-sided, with $\mathrm{p}<0.05$ defined as statistically significant.

To avoid potential biases associated with the use of standard survival analysis in this context, interval-censored data analysis methods were used (see supplementary methods section). The multivariate analysis was performed by comparisons between haplogroups considering the most common haplogroup $\mathrm{H}$ as the reference group. Since there was no interest in all possible pairwise comparisons, no additional adjusting for multiple comparisons was done.

\section{Meta-analysis}

In this work, we performed a meta-analysis of incident knee OA including data from CHECK and OAI. Following the Preferred Reporting Items for Systematic Reviews and Meta-analyses (PRISMA) guidelines, we conducted a previous computerised search strategy to find other possible relevant studies to include in the meta-analysis (see supplementary methods).

The random-effects model described by DerSimonian and Laird $^{26}$ was used to calculate a summary statistic and its $95 \%$
CI. Adjusted HRs were used as the effect size measure for the association between mtDNA haplogroups and $\mathrm{OA}$ incidence. Meta-analysis results were presented on a forest plot graph. To explore heterogeneity, the $\mathrm{I}^{2}$ index was computed. Meta-analysis was carried out using the R software programme (V.3.2.2), using the meta-package. A two-tailed $p$ value $<0.05$ was considered to be significant. Meta-analysis was planned to be performed on $\mathrm{k}=2$ studies, with an estimated statistical power of $91.9 \%$ to detect as statistically significant an HR $\leq 0.5$ associated with each haplogroup, with a $\mathrm{p}=0.05$ two-tailed significance level.

We only selected studies that met the following inclusion criteria: (i) evaluating the association between mtDNA haplogroups and the rate of incident OA over time; (ii) with sufficient data provided to calculate HRs with their corresponding 95\% CI. On the contrary, those studies analysing the correlation between mtDNA haplogroups and the prevalence or risk of OA as well as those studies analysing other mtDNA mutations with incidence were excluded.

\section{Functional studies using transmitochondrial cybrids}

An extended version of the methodology described herein is available in the online supplementary methods.

\section{Cybrid cultures and culture conditions}

The human osteosarcoma cell line 143B.TK ${ }^{-}$Rho-0 was used to generate the transmitochondrial cybrids by fusing them with platelets from healthy donors carrying the haplogroups $\mathrm{J}$ or $\mathrm{H}$ following the protocol described by Chomyn, ${ }^{27}$ such that we finally obtained two types of cybrids: cybrids harbouring haplogroup J and cybrids harbouring haplogroup $\mathrm{H}$.

\section{Flux assay measurements}

Extracellular acidification rate (ECAR) (largely result of glycolysis) and oxygen consumption rate (OCR) (indicator of mitochondrial respiration) was determined by direct measurement in a SeaHorse XFp extracellular flux analyser instrument (Seahorse Bioscience, Agilent Technologies) following manufacturer's instructions.

\section{Mitochondrial reactive oxygen species production assay}

Mitochondrial peroxide and peroxynitrite were evaluated with dihydrorhodamine 123 (Sigma) and mitochondrial superoxide anion production was assessed with MitoSox Red (Thermo Fisher Scientific).

\section{Oxidative stress response assay: viability assay}

After incubation of cells with $300 \mu \mathrm{M} \mathrm{H}_{2} \mathrm{O}_{2}$, the cell viability was measured using CellTiter 96 Aqueous Assay (Promega) following manufacturer's recommendations.

\section{Apoptosis assay}

Cells were cultured in presence of staurosporine at $0.2 \mu \mathrm{M}$ during 2 hours to induce apoptosis and were subsequently washed and resuspended in $1 \mathrm{X}$ Annexin-binding buffer; then $5 \mu \mathrm{L}$ of the Annexin V-fluorescein isothiocyanate and $5 \mu \mathrm{L}$ of propidium iodide (ImmunoStep) were added to each $100 \mu \mathrm{L}$ of cell suspension.

In addition, the basal expression of the mitochondrial apoptotic-related genes B-cell CLL/lymphoma 2-like 13 (BCL2L13) and BCL2 binding component 3 (BBC3) in $\mathrm{H}$ and $\mathrm{J}$ cybrids was also quantified. 


\section{Statistical analysis}

Results were expressed as the mean of three independent experiments (mean $\pm S D$ ) using two cybrids (two J cybrids and two $\mathrm{H}$ cybrids) from two individuals (two different $\mathrm{J}$ individuals and two different $\mathrm{H}$ individuals) and two clones from each cybrid. Statistically significant differences between the two groups were determined with t-test; $\mathrm{p}$ values $<0.05$ were considered to be significant.

\section{RESULTS \\ mtDNA haplogroups and incident knee OA in subjects of the OAl cohort}

No significant differences were detected in the distribution of age, BMI, Western Ontario and McMaster Universities Arthritis Index (WOMAC) and contralateral $\mathrm{OA}$ at baseline among patients with different haplogroups; however, a significantly different gender distribution was identified, both being haplogroups $\mathrm{H}$ and $\mathrm{J}$ that showed an increased percentage of females than the rest of haplogroups (table 1).

Cumulative probability of incident knee OA at 8 years was $25.1 \%$. Subjects with the haplogroup $\mathrm{J}$ showed the lowest rate of incident knee OA over time, 20.1\% (adjusted $\mathrm{HR}=0.680$; $95 \%$ CI 0.470 to $0.968 ; \mathrm{p}<0.05)$. In addition, males had a decreased risk too (adjusted $\mathrm{HR}=0.590 ; 95 \% \mathrm{CI} 0.491$ to 0.712; p <0.05), meanwhile BMI (adjusted HR $=1.066 ; 95 \% \mathrm{CI}$ 1.047 to $1.085 ; \mathrm{p}<0.05$ ), total WOMAC (adjusted $\mathrm{HR}=1.018$; $95 \%$ CI 1.010 to $1.026 ; \mathrm{p}<0.05)$ and contralateral knee OA (adjusted $\mathrm{HR}=1.593 ; 95 \%$ CI 1.328 to $1.900 ; \mathrm{p}<0.05$ ) at baseline were risk factors for a higher risk of incident knee OA (table 2).

\section{mtDNA haplogroups and incident knee $O A$ in subjects of CHECK cohort}

No significant differences were detected in the distribution of age, gender, BMI, WOMAC and contralateral OA at baseline among patients with different haplogroups (table 1).

Global cumulative probability of incident knee OA at 8 years was $89.7 \%$. The cumulative knee OA incidence was significantly lower in carriers of the haplogroup $\mathrm{J}$, with $82.1 \%$ (adjusted $\mathrm{HR}=0.728 ; 95 \%$ CI 0.469 to $0.998 ; \mathrm{p}<0.05)$. In addition, the model also showed that total WOMAC (adjusted HR=1.006; $95 \%$ CI 1.001 to $1.012 ; \mathrm{p}<0.05$ ) and contralateral knee OA (adjusted HR $=1.313$; 95\% CI 1.087 to 1.576 ; $<<0.05$ ) at baseline were risk factors to develop incident knee OA (table 2).

\section{Meta-analysis}

The search process identified a total of seven non-duplicated articles and no unpublished article $\begin{array}{lllll}16 & 18 & 22 & 28-31 & \text { (see online }\end{array}$ supplementary table S1). However, after reading the titles and abstracts none of these seven articles was subsequently selected for meta-analysis because they did not follow the inclusion criteria; namely, they did not evaluate the association between mtDNA haplogroups and the rate of incident knee OA over time but analysed the association between these mtDNA variants, or any other mtDNA mutations, and the prevalence of OA in cross-sectional studies. In conclusion, only the two association studies performed herein were selected for subsequent meta-analysis reaching a total of 3214 subjects (2579 patients from the OAI and 635 patients from CHECK) (table 3).

No between-study heterogeneity was detected for the haplogroup J $\left(\mathrm{I}^{2}=0 \% ; \mathrm{p}=0.798\right)$, however, the random-effects model was evenly used. Both studies showed a similar contribution or relative weight (W), being slightly higher in the OAI

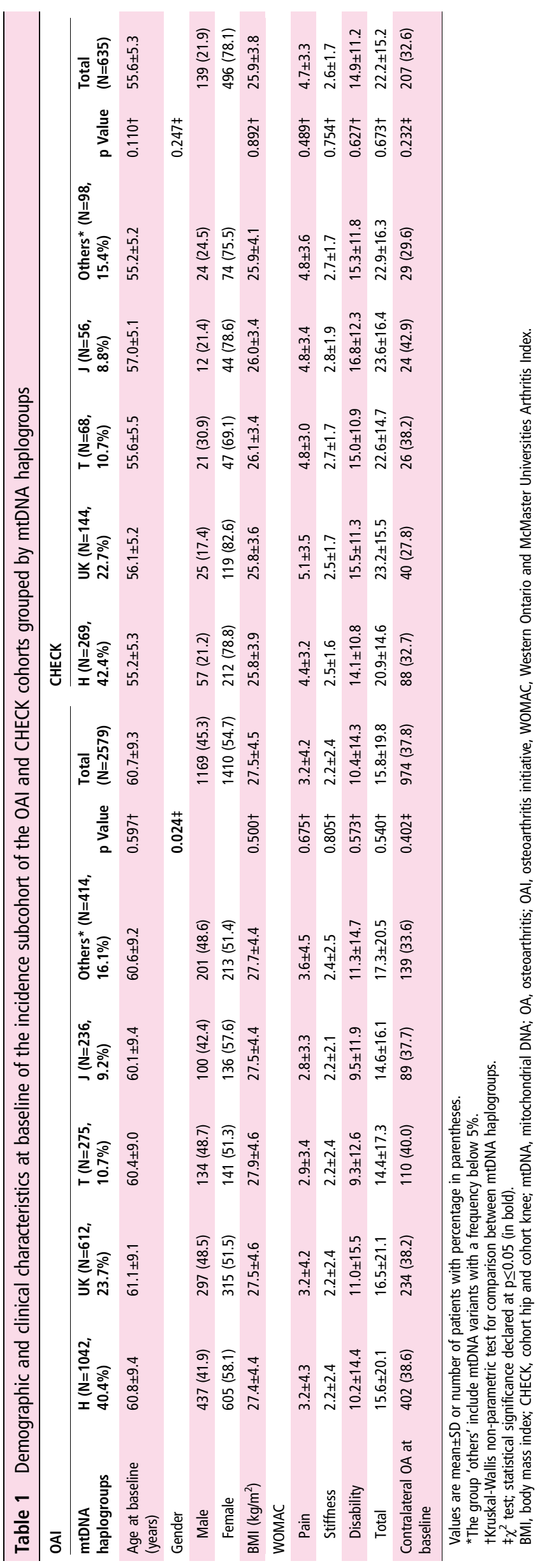


Table 2 Cumulative probability of incident knee OA at 8 years in subjects from the OAI and CHECK cohorts according to mtDNA haplogroups and results of the extended Cox proportional hazard model

\begin{tabular}{|c|c|c|c|}
\hline Variables & $\begin{array}{l}\text { Cumulative knee } \mathrm{OA} \text { incidence at } 8 \text { years } \\
\mathrm{n}(\%)^{*}\end{array}$ & Adjusted HR & $95 \% \mathrm{Cl} t$ \\
\hline \multicolumn{4}{|l|}{ OAI } \\
\hline Gender (male) & & 0.590 & 0.491 to $0.712 \ddagger$ \\
\hline Age & & 1.007 & 0.997 to 1.017 \\
\hline BMI & & 1.066 & 1.047 to $1.085 \ddagger$ \\
\hline WOMAC (total) & & 1.018 & 1.010 to $1.026 \ddagger$ \\
\hline Contralateral $\mathrm{OA}$ at baseline & & 1.593 & 1.328 to $1.900 \ddagger$ \\
\hline \multicolumn{4}{|l|}{ mtDNA haplogroups $(\mathrm{N}=2579)$} \\
\hline$H(n=1042)$ & $278(26.7 \%)$ & 1 & \\
\hline UK $(n=612)$ & $154(25.1 \%)$ & 0.908 & 0.727 to 1.131 \\
\hline$T(n=275)$ & $67(24.3 \%)$ & 0.896 & 0.658 to 1.221 \\
\hline$J(n=236)$ & $47(20.1 \%)$ & 0.680 & 0.470 to $0.968 \ddagger$ \\
\hline Others§ $(n=414)$ & $102(24.6 \%)$ & 0.895 & 0.698 to 1.153 \\
\hline \multicolumn{4}{|l|}{ CHECK } \\
\hline Gender (male) & & 0.869 & 0.698 to 1.043 \\
\hline Age & & 1.007 & 0.990 to 1.026 \\
\hline BMI & & 1.005 & 0.987 to 1.025 \\
\hline WOMAC (total) & & 1.006 & 1.001 to $1.012 \ddagger$ \\
\hline Contralateral $\mathrm{OA}$ at baseline & & 1.313 & 1.087 to $1.576 \ddagger$ \\
\hline \multicolumn{4}{|l|}{ mtDNA haplogroups ( $\mathrm{N}=635)$} \\
\hline$H(n=269)$ & $248(92.2 \%)$ & 1 & \\
\hline UK $(n=144)$ & $126(87.5 \%)$ & 0.849 & 0.682 to 1.035 \\
\hline$T(n=68)$ & $64(94.1 \%)$ & 1.133 & 0.850 to 1.447 \\
\hline$J(n=56)$ & $46(82.1 \%)$ & 0.728 & 0.469 to $0.998 \ddagger$ \\
\hline Others§ ( $n=98)$ & $86(87.7 \%)$ & 0.810 & 0.627 to 1.032 \\
\hline
\end{tabular}

${ }^{*}$ Cumulative incident knee OA rate from baseline to follow-up.

tCls for the HRs obtained using the bootstrap methodology by the improved percentile method.

$¥$ Statistical significance declared at $p \leq 0.05$.

$\S$ The group 'others' include mtDNA variants with a frequency below $5 \%$.

Bold refers to parameters that reached the statistical significance $(p \leq 0.05)$

BMI, body mass index; CHECK, cohort hip and cohort knee; mtDNA, mitochondrial DNA; OA, osteoarthritis; OAI, osteoarthritis initiative, WOMAC, Western Ontario and McMaster Universities Arthritis Index.

Table 3 Characteristics of studies included in the meta-analysis

\begin{tabular}{|c|c|c|c|c|c|c|c|c|c|c|c|}
\hline Cohort & Year & $\begin{array}{l}\text { Sample } \\
\text { size }\end{array}$ & Country & Ethnicity & $\begin{array}{l}\text { Mean } \\
\text { age }\end{array}$ & Type of study & $\begin{array}{l}\text { Type } \\
\text { of } O A\end{array}$ & $\begin{array}{l}\text { Incident } \\
\text { knee OA } \\
\text { criteria }\end{array}$ & $\begin{array}{l}\text { Genotyping } \\
\text { method }\end{array}$ & $\begin{array}{l}\text { Controlled } \\
\text { confounder } \\
\text { variables }\end{array}$ & Conclusion \\
\hline CHECK & 2016 & 635 & $\begin{array}{l}\text { The } \\
\text { Netherlands }\end{array}$ & Caucasian & 56 & $\begin{array}{l}\text { Incidence, } \\
\text { prospective at } 8 \\
\text { years }\end{array}$ & $\begin{array}{l}\text { Knee } \\
\text { OA }\end{array}$ & $\begin{array}{l}\text { New-onset } \mathrm{KL} \\
\text { grade } 1\end{array}$ & $\begin{array}{l}\text { SBE and PCR/ } \\
\text { RFLP }\end{array}$ & $\begin{array}{l}\text { Gender, age, BMI, } \\
\text { WOMAC, } \\
\text { contralateral OA }\end{array}$ & $\begin{array}{l}\text { Haplogroup J } \\
\text { associates with } \\
\text { decreased risk }\end{array}$ \\
\hline OAI & 2016 & 2579 & USA & Caucasian & 61 & $\begin{array}{l}\text { Incidence, } \\
\text { Prospective at } \\
\text { eight years }\end{array}$ & $\begin{array}{l}\text { Knee } \\
\text { OA }\end{array}$ & $\begin{array}{l}\text { New-onset KL } \\
\text { grade } 2\end{array}$ & $\begin{array}{l}\text { SBE and PCR/ } \\
\text { RFLP }\end{array}$ & $\begin{array}{l}\text { Gender, age, BMI, } \\
\text { WOMAC, } \\
\text { contralateral OA }\end{array}$ & $\begin{array}{l}\text { Haplogroup J } \\
\text { associates with } \\
\text { decreased risk }\end{array}$ \\
\hline
\end{tabular}

BMI, body mass index; CHECK, cohort hip and cohort knee; KL, Kellgren and Lawrence; OA, osteoarthritis; SBE, single base extension; OAl, osteoarthritis initiative; RFLP, restriction fragment length polymorphism; WOMAC, Western Ontario and McMaster Universities Arthritis Index.

study (52.2\%) compared with CHECK (47.8\%). The results showed that haplogroup $\mathrm{J}$ significantly reduces the risk of incident knee OA (combined $\mathrm{HR}=0.702$; $95 \%$ CI 0.541 to 0.912 ; $\mathrm{p}=0.008)$ (figure 1).

\section{Functional study using transmitochondrial cybrids}

Establishment of basal conditions

At 25 passages, the mtDNA copy number between $\mathrm{H}$ and $\mathrm{J}$ cybrids acquired the steady state levels (data not shown), meanwhile at 48 hours the doubling time (DT) showed no significant differences between $\mathrm{H}$ and $\mathrm{J}$ cybrids (data not shown). Based on these setting, all the experiments were performed starting from 25 passages and after 48 hours of cell culture.

Mitochondrial respiration and glycolytic capacity

To measure real-time glycolytic and mitochondrial respiration rates, cybrids were placed in an extracellular flux analyser (Seahorse Biosciences). The analyser measures ECAR and OCR in a transient microchamber, representing glycolysis and mitochondrial respiration, respectively. Glycolysis rate in $\mathrm{H}$ and $\mathrm{J}$ cybrids were $87.98 \pm 11.4$ and $69.45 \pm 4.69 \mathrm{mpH} / \mathrm{min}$, respectively, indicating a $21.06 \%$ decrease in J cybrids $(\mathrm{p}=0.0004)$; 


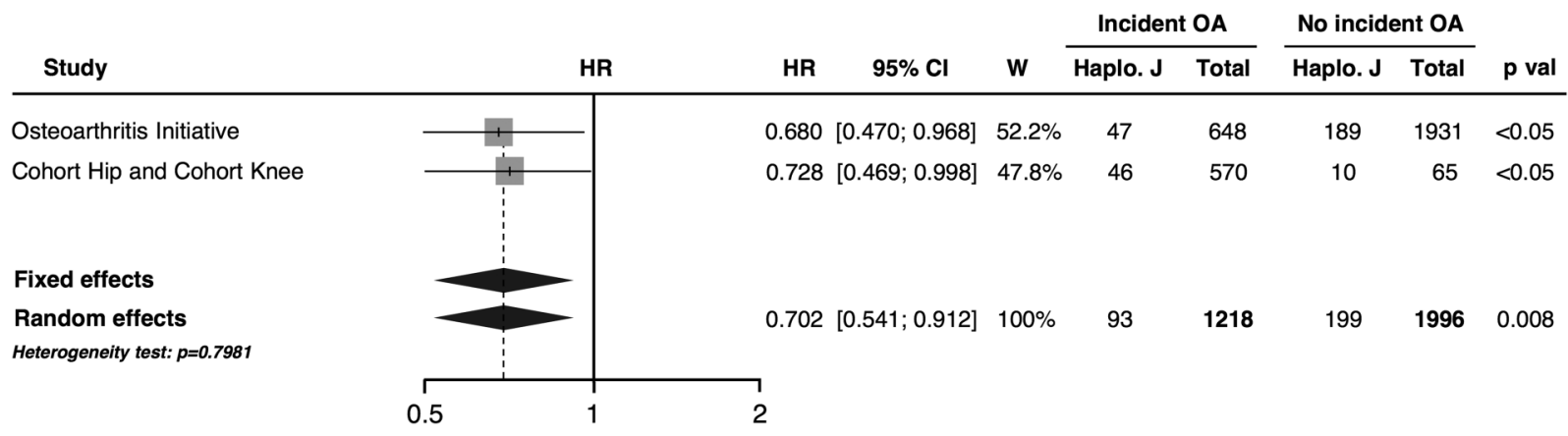

Figure 1 Forest plot of the associations analysed separately in this work involving the mitochondrial DNA haplogroup J and the risk of incident knee OA. OA, osteoarthritis; OAI, osteoarthritis initiative; CHECK, cohort hip and cohort knee; W, relative weight; Haplo. J, number of subjects with the haplogroup $J$ that developed and did not develop incident knee OA during the follow-up; $p$ Val: $p$ Value.

glycolytic capacity rates in $\mathrm{H}$ and $\mathrm{J}$ cybrids were $116.3 \pm 12.76$ and $91.69 \pm 6.48 \mathrm{mph} / \mathrm{min}$, respectively, indicating a $21.16 \%$ decrease in J cybrids $(\mathrm{p}<0.0001)$ (figure $2 \mathrm{~A})$. Similarly, mitochondrial basal respiration rate was significantly lower in $\mathrm{J}$ cybrids $(96.58 \pm 60.01 \mathrm{pmol} / \mathrm{min})$ compared with $\mathrm{H}$ cybrids $(209.08 \pm 55.7 \mathrm{pmol} / \mathrm{min}, \mathrm{p}=0.007)$; as a result, $\mathrm{H}$ cybrids showed an increased ATP production $(149.95 \pm 23.29$ vs 65.94 $\pm 25.72 \mathrm{pmol} / \mathrm{min}$ in J cybrids; $\mathrm{p}<0.0001$ ) (figure $2 \mathrm{~B}$ ).

\section{Mitochondrial reactive oxygen species production and oxidative stress response}

Cybrids carrying the haplogroup $\mathrm{H}$ had a significantly higher production of peroxide and peroxynitrite than cybrids carrying the haplogroup J $(52.51 \pm 11.34$ vs $41.26 \pm 7.48 ; \mathrm{p}<0.05)$ (figure 2C). Moreover, the analysis of mitochondrial anion superoxide revealed the same significant trend by which $\mathrm{H}$ cybrids showed higher levels than J cybrids $(8.58 \pm 3.0$ vs 4.25 $\pm 0.9 ; \mathrm{p}<0.05$ ) (figure 2D).

In relation to oxidative stress response, the results showed that the percentage of survival cells in presence of $\mathrm{H}_{2} \mathrm{O}_{2}$ in $\mathrm{H}$ cybrids was significantly lower than in J cybrids $(29.63 \pm 3.3$ vs $56.45 \pm 7.36 ; \mathrm{p}<0.05$ ) (figure 2E).

\section{Analysis of apoptosis}

The analysis of apoptosis assessed in basal conditions revealed no significant differences between $\mathrm{H}$ and J cybrids $(3.69 \pm 2$ and $4.17 \pm 1.1$, respectively; $\mathrm{p}>0.05$ ) (data not shown). However, after incubation with staurosporine, cybrids carrying the haplogroup $\mathrm{H}$ showed a significantly increased number of apoptotic cells in comparison with $\mathrm{J}$ cybrids $(7.35 \pm 3.78$ vs $4.69 \pm 1.68$; $\mathrm{p}<0.05$ ) (figure $2 \mathrm{~F}$ ).

Further analysis of the pro-apoptotic gene BBC3 and the antiapoptotic gene BCL2L13 showed a significantly increased expression of $\mathrm{BBC} 3$ in $\mathrm{H}$ cybrids compared with $\mathrm{J}$ cybrids $(3.3$-fold; $\mathrm{p}<0.05)$. On the contrary, no significant differences were detected in the expression of BCL2L13 (figure 2G).

\section{DISCUSSION}

Here, we report the first replication study followed by a meta-analysis addressing the association between mtDNA haplogroups and the risk of incident knee OA over time using new haplogroup data from two well-characterised cohorts of patients; a previous meta-analysis involving the study of the mtDNA haplogroups consisted in the analysis of their association with the prevalence of the disease. ${ }^{28}$ In addition, we provide data supporting the possible explanation for these associations using transmitochondrial cybrids.
We assessed, for the first time, the influence of the mtDNA haplogroups in the rate of incident knee OA in subjects of the incidence subcohort of the OAI followed by a replication study in patients from CHECK. Despite OAI and CHECK are geographically different cohorts, the frequency distribution of the mtDNA haplogroups was very similar between both cohorts $\left(\chi^{2}\right.$ test; $p=0.924$, data not shown), ruling out a potential confusion due to ethnic origin. Compared with the incidence subgroup of the OAI, CHECK participants experienced an increased radiographic change at follow-up (89.7\% vs $25.1 \%$ ) mainly because of the different incident knee OA criteria and mean age at baseline between both cohorts, as previously described. ${ }^{32}$ Although both cohorts focus on the early phase of $\mathrm{OA}$, the CHECK cohort represents participants in an even earlier state of the disease $\mathrm{e}^{33}$ and the incident knee OA criteria slightly differs from the proposed by Felson et $a l^{23}$ in subjects of the OAI. Notwithstanding, the results obtained reveal that subjects with haplogroup $\mathrm{J}$ show a lower rate of incident knee OA in both cohorts.

To date, different associations involving the haplogroup J have been described in the context of OA. Subjects carrying this haplogroup have a lower prevalence of knee and/or hip OA, ${ }^{16} 171928$ besides this mtDNA variant also associates with lower serum levels of catabolic type II collagen biomarkers ${ }^{34}$ and metalloproteinases (MMPs) ${ }^{35}$ and has been correlated with higher telomere length and lower nitric oxide production in articular chondrocytes. ${ }^{36}$ However, a work by Hudson et al found no evidence of associations between mtDNA variants and the risk of OA. ${ }^{20}$ Although the study by Hudson $e$ al is a prevalence study, and not an incident knee OA analysis as is the case of the study presented herein, some points could clarify this; control samples used in their study are population-based controls with symptomatic information and without radiographic data. Up to $50 \%$ of patients without joint symptoms may have radiographic changes related to $\mathrm{OA},{ }^{37}$ therefore, the selection of adequate healthy controls is crucial to draw consistent conclusions in case-control studies; this point could be one of the causes by which one study performed by the arcOGEN consortium also failed to replicate previous associations at genomewide significance level $\left(\mathrm{p} \leq 5.0 \times 10^{-8}\right)$, such as GDF5, chromosome $7 \mathrm{q} 22$ or MCF2L gene polymorphisms. ${ }^{38}$ Finally, as postulated by these authors, the relative contribution of specific mtDNA variants could vary in different ethnic groups by means of homoplasy and/or geographic differences in the finer details of subhaplogroup structures of mtDNA. ${ }^{20}$

The results of the meta-analysis indicate that the associations are robust and reveal a strong association with the haplogroup $\mathrm{J}$. The haplogroup $\mathrm{J}$ is characterised by a set of uncoupling 
A

ECAR

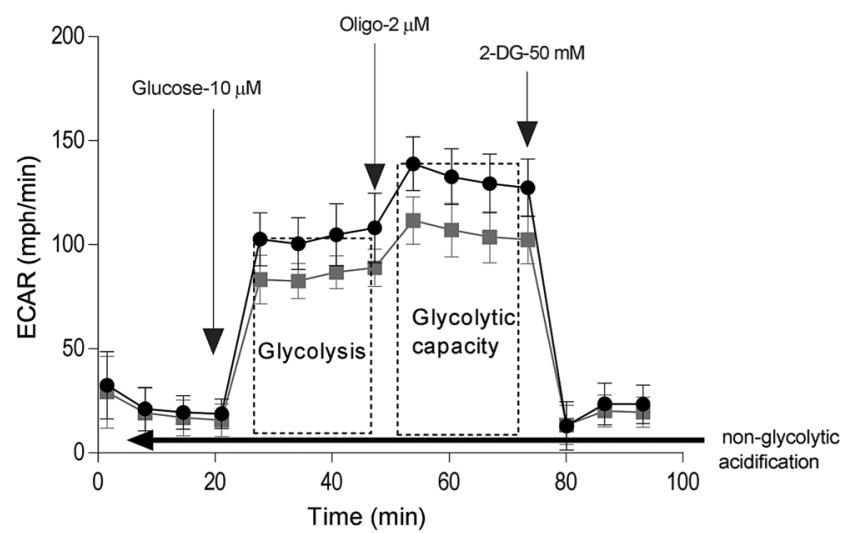

B

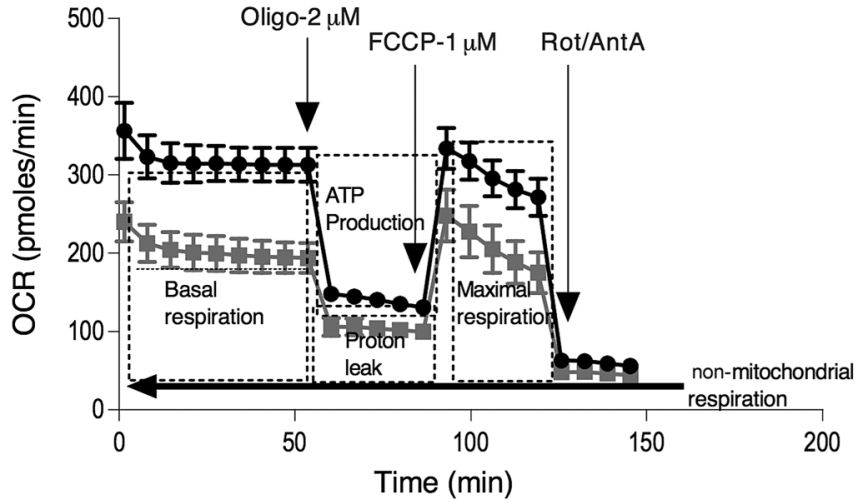

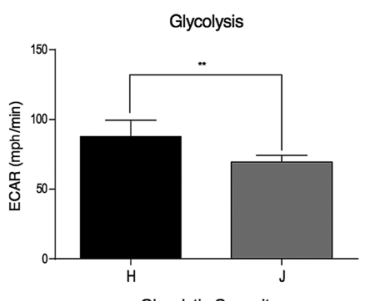

Glycolytic Capacity
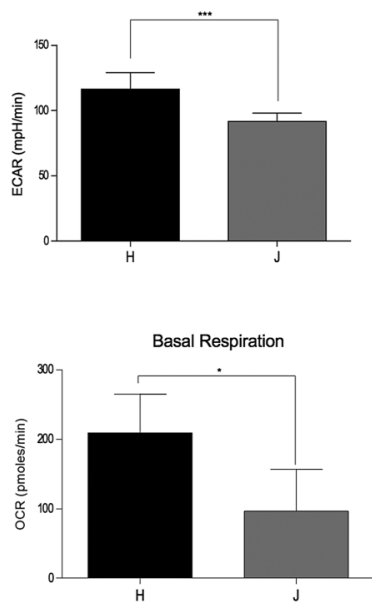

ATP production
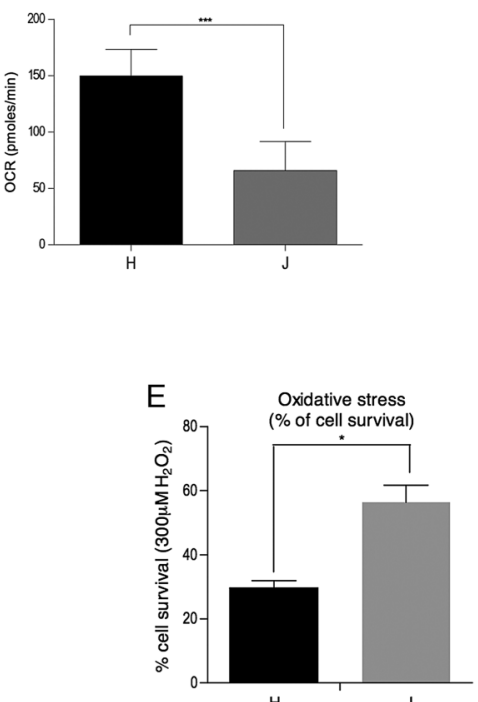

C

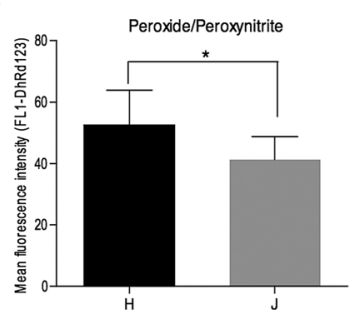

D

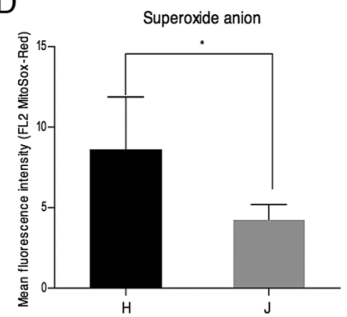

Figure 2 (A) Glycolysis stress test by extracellular acidification rate (ECAR) traces and bar graphs showing the glycolytic response of $\mathrm{H}$ and $\mathrm{J}$ cybrids in response to glucose, oligomycin (oligo) and 2-deoxy-glucose (2-DG) injection where indicated. Bar graphs show glycolysis and glycolytic capacity as calculated from trace and compared between groups. (B) Mitochondrial stress test by OCR traces and bar graphs showing the mitochondrial behaviour in $\mathrm{H}$ and $\mathrm{J}$ cybrids in response to oligomycin, FCCP and rotenone/antimycin (Rot/AntA) injection where indicated. Bar graphs show basal respiration and ATP production, calculated and compared between groups; (C) mitochondrial peroxide and peroxynitrite production; (D) mitochondrial anion superoxide production; (E) susceptibility to oxidative stress after incubation with $300 \mu \mathrm{M} \mathrm{H} \mathrm{O}_{2}$; (F) apoptosis measure with Annexin-V-fluorescein isothiocyanate: data are expressed as percentage of positive cells under stress induction with staurosporine at $0.2 \mu \mathrm{M}$; (G) gene expression of the apoptotic genes BBC3 and BCL2. The values represented were a mean $\pm S D$ of three independent experiments using two cybrids from two different individuals and two clones from each cybrid. Black bars corresponds to $\mathrm{H}$ cybrids, while grey bars correspond to J cybrids; BBC3, BCL2 binding component; FCCP, carbonyl cyanide $p$-trifluoromethoxyphenylhydrazone; ${ }^{* * *} p<0.0001 ;{ }^{* *} p<0.01 ;{ }^{*} p<0.05$. 
mitochondrial polymorphisms, ${ }^{9} 10$ preferentially non-synonymous SNPs, ${ }^{39}$ acquired during its evolutionary history. These aspects make this haplogroup to be biochemically different from other mitochondrial variants, specially the haplogroup $\mathrm{H}^{40} 41$

Increasing evidence suggests that low-grade chronic inflammation in the joint promotes OA progression, and changes in cellular bioenergy metabolism can reprogramme inflammatory response, leading to the disturbance of cellular energy balance and increase cell stress. ${ }^{42}$ This evidence implies that mitochondria, as the regulators of cell metabolism, as well as the mtDNA haplogroups, as one of the main modulators of cellular bioenergetics, ${ }^{43}$ are involved in the development of OA as previously proposed. $^{2}{ }^{44}$ Nevertheless, in an attempt to find out the possible cellular mechanisms underlying the associations described herein, we performed a functional study of the mtDNA haplogroups using transmitochondrial cybrids.

Transmitochondrial cybrids are cell lines consisting of mitochondria from different sources in a defined and uniform nuclear background. They constitute an interesting model and allow the study of the real role of different mtDNA polymorphisms under identical nuclei, and they also provide a window into early stages of disease pathogenesis, which is not available from pathological specimens. Because of that, these cell models have been proposed and widely used to explore the contribution of mitochondrial dysfunction and mtDNA mutations to the pathogenesis of human diseases, such as Parkinson or cancer. $^{45}{ }^{46}$ Through the use of this cellular model, recent studies showed that $\mathrm{J}$ variant associates with decreased expression of specific genes related to inflammatory response, complement and apoptosis when compared with the haplogroup H. ${ }^{12}$ The functional analysis described herein included specific metabolic measurements using an extracellular flux analyser as well as specific aspects proposed to be related to OA, such as mitochondrial reactive oxygen species (ROS) production, oxidative stress and apoptosis. ${ }^{74} 47$ The results obtained reveal that cells harbouring the haplogroup $\mathrm{J}$ show a physiological behaviour that seems to be protective against the development of OA.

This physiological behaviour includes a significantly lower production of mitochondrial superoxide anion and peroxynitrite, as well as a higher ability to cope with oxidative stress. It has been demonstrated that mitochondria-derived ROS and nitrogen radicals lead to an upregulation of $\mathrm{MMPs}^{44}$ as well as an overproduction of pro-inflammatory cytokines, ${ }^{48}$ cellular damage or, in some cases, apoptosis. Interestingly, cybrids harbouring the haplogroup J show a significant lower rate of apoptosis under stress conditions, and a lower expression of the pro-apoptotic gene $\mathrm{BBC} 3$, which induces apoptosis through mitochondrial dysfunction.

From a metabolic point of view, haplogroups $\mathrm{H}$ and $\mathrm{J}$ have a different behaviour too. $\mathrm{H}$ cybrids show higher mitochondrial respiration rate and glycolytic capacity, which is reflected in an increased ATP generation compared with cybrids carrying the haplogroup J. The haplogroup $\mathrm{H}$ presents the highest levels of conserved amino acids, ${ }^{9}$ which could determine its Oxidative Phosphorylation System (OXPHOS) coupling efficiency and ATP production $^{41}$; however, this high efficiency would be accompanied by an increased ROS generation and a higher oxygen consumption, as demonstrated herein and proposed elsewhere. ${ }^{10} 41$

In recent years, several studies supported a key role of the mitochondria in the pathogenesis of OA and the study of this organelle in the context of this disease attracted much attention. $^{2} 4950$ The mechanisms underlying, at least in part, the association described herein are related to different functional consequences characteristic of specific mitochondrial polymorphisms; thus, ROS production (both peroxynitrite and superoxide anion), oxidative stress and apoptosis are downregulated in cells harbouring mitochondrial polymorphisms characteristic of the mtDNA haplogroup J.

Although a direct functional link between haplogroups and cartilage biology has not been made in this study, a recent work in conplastic mice (mice with constant nuclear background but different mtDNA variants) shows that mtDNA haplotype profoundly influences in health longevity through mitochondrial proteostasis and ROS generation, insulin signalling, obesity, telomere shortening or mitochondrial dysfunction. ${ }^{51}$ Because most of these aspects are also involved in OA pathogenesis, a functional link between mtDNA variation and cartilage biology could really exist.

Despite only two studies were combined, the results of the meta-analysis are consistent enough to support a real association of haplogroup $\mathrm{J}$ with a lower risk of incident knee OA. Furthermore, data from both cohorts were analysed in a similar way, and effect size measures were adjusted for baseline characteristics in order to minimise residual confounding. Both OAI and CHECK are well-characterised cohorts, constructed with rigorous methodology in which patients are evaluated by objective methods in spaced visits. This should contribute to minimise information biases and strengthen the conclusions of this study.

In summary, the results of this work have a special clinical relevance. On the one hand, they claim that polymorphisms of the mtDNA haplogroup J alter metabolism and cell physiology predisposing the cell to a less favourable environment to develop incident $\mathrm{OA}$, which allows the design of potential drugs that emulate the physiological effects related to this haplogroup, as well as the consideration of the mtDNA haplogroups as candidate diagnostic biomarkers in OA; among these therapeutic strategies, potential drugs that modulate the activity of the mitochondrial respiratory chain in a similar way to that of the haplogroup J, or even the development of a cellular therapy using cells with mitochondria harbouring the haplogroup J, could be interesting. On the other hand, the results obtained permit to select patients with OA not harbouring the haplogroup J (ie, haplogroup $\mathrm{H}$ ) as ideal candidates for clinical trials because they are more likely to suffer a higher rate of incident knee OA.

\begin{abstract}
Author affiliations
${ }^{1}$ Servicio de Reumatología, Instituto de Investigación Biomédica de A Coruña (INIBIC), Complexo Hospitalario Universitario de A Coruña (CHUAC), Sergas, Universidade da Coruña (UDC), As Xubias, A Coruña, España

2Plataforma de Genómica, Instituto de Investigación Biomédica de A Coruña (INIBIC), Complexo Hospitalario Universitario de A Coruña (CHUAC), Sergas, Universidade da Coruña (UDC), As Xubias, A Coruña, España

${ }^{3}$ Unidad de Epidemiología Clínica y Bioestadística, Instituto de Investigación Biomédica de A Coruña (INIBIC), Complexo Hospitalario Universitario de A Coruña (CHUAC), Sergas, Universidade da Coruña (UDC), As Xubias, A Coruña, España ${ }^{4}$ Departamento de Bioquímica, Instituto de Investigaciones Biomédicas "Alberto Sols" UAM-CSIC y Centro de Investigación Biomédica en Red de Enfermedades Raras (CIBERER), Universidad Autónoma de Madrid, Madrid, Spain

${ }^{5}$ Laboratorio de Enfermedades Mitocondriales, Instituto de Investigación Sanitaria Hospital 12 de Octubre (i+12) Madrid, Madrid, Spain
\end{abstract}

\section{Twitter Follow Ignacio Rego-Perez at @nacho_rego}

Acknowledgements The OAI is a public-private partnership comprising five contracts (N01-AR-2-2258; N01-AR-2-2259; N01-AR-2-2260; N01-AR-2-2261; N01-AR-2-2262) funded by the National Institutes of Health (NIH), a branch of the Department of Health and Human Services, and conducted by the OAI Study Investigators. Private funding partners include Pfizer; Novartis Pharmaceuticals; Merck Research Laboratories and GlaxoSmithKline. Private sector funding for the OA is managed by the Foundation for the NIH. This manuscript was prepared using an OAl public use data set and does not necessarily reflect the opinions or views of the $\mathrm{OAl}$ investigators, the $\mathrm{NIH}$ or the private funding partners. CHECK cohort study is 
funded by the Dutch Arthritis Association. It is led by a steering committee comprising 16 members with expertise in different fields of OA. It is chaired by Professor J W J Bijlsma and coordinated by J Wesseling. The following institutions are involved: Erasmus Medical Center Rotterdam; Kennemer Gasthuis Haarlem; Leiden University Medical Center; Maastricht University Medical Center; Martini Hospital Groningen/Allied Health Care Center for Rheumatology and Rehabilitation Groningen; Medical Spectrum Twente Enschede/Ziekenhuisgroep Twente Almelo; Reade, formerly Jan van Breemen Institute/VU Medical Center Amsterdam; St Maartens-kliniek Nijmegen; University Medical Center Utrecht and Wilhelmina Hospital Assen. We would like to thank the participants, principal investigators, co-investigators and staff of CHECK.

Contributors FJB and IR-P contributed equally in the design and coordination of the study; both conceived the study, participated in its design and helped to draft the final version of the manuscript; MF-M carried out the cybrid experiments and helped to draft the manuscript and data interpretation; AS-H carried out the experimental procedures of mitochondrial haplogroup assignment; MEV-M, EC-P, SR and TH-G helped to carry out the cybrids cell culture, the subsequent experimental procedures and the haplogroup assignment; SP supervised the statistical procedures; NO-V and CF-L helped the understanding of the clinical and radiological variables included in the different OAI and CHECK datasets; RG provided the necessary infrastructure for the development of the transmitochondrial cybrids. All the authors approved the final version of the manuscript.

Funding This study was supported by grants from Fondo de Investigación Sanitaria CIBERCB06/01/0040-Spain, RETIC-RIER-RD12/0009/0018 and PI14/01254, integrated in the National Plan for Scientific Program, Development and Technological Innovation 2013-2016 and funded by the ISCIII-General Subdirection of Assessment and Promotion of Research-European Regional Development Fund (FEDER) 'A way of making Europe'. IRP is supported by Contrato Miguel Servet-Fondo de Investigación Sanitaria (CP12/03192).

Competing interests None declared.

Patient consent Obtained.

Ethics approval National Institutes of Health and Dutch Arthritis Association.

Provenance and peer review Not commissioned; externally peer reviewed.

Open Access This is an Open Access article distributed in accordance with the Creative Commons Attribution Non Commercial (CC BY-NC 4.0) license, which permits others to distribute, remix, adapt, build upon this work non-commercially, and license their derivative works on different terms, provided the original work is properly cited and the use is non-commercial. See: http://creativecommons.org/ licenses/by-nc/4.0/

\section{REFERENCES}

1 Kraus VB, Blanco FJ, Englund $\mathrm{M}$, et al. Call for standardized definitions of osteoarthritis and risk stratification for clinical trials and clinical use. Osteoarthr Cartil 2015:23:1233-41.

2 Blanco FJ, Rego I, Ruiz-Romero C. The role of mitochondria in osteoarthritis. Nat Rev Rheumatol 2011:7:161-9.

3 Felson D, Niu J, Sack B, et al. Progression of osteoarthritis as a state of inertia. Ann Rheum Dis 2013:72:924-9.

4 Minafra L, Bravatà V, Saporito $M$, et al. Genetic, clinical and radiographic signs in knee osteoarthritis susceptibility. Arthritis Res Ther 2014;16:R91.

5 Takahashi H, Nakajima M, Ozaki K, et al. Prediction model for knee osteoarthritis based on genetic and clinical information. Arthritis Res Ther 2010;12:R187.

6 Blanco FJ, Möller I, Romera M, et al. Improved prediction of knee osteoarthritis progression by genetic polymorphisms: the Arthrotest Study. Rheumatology 2015:54:1236-43.

7 Wang $Y$, Zhao $X$, Lotz $M$, et al. Mitochondrial biogenesis is impaired in osteoarthritis chondrocytes but reversible via peroxisome proliferator-activated receptor $\gamma$ coactivator $1 \alpha$. Arthritis Rheumatol 2015;67:2141-53.

8 Fernandez-Moreno M, Soto-Hermida A, Oreiro N, et al. Mitochondrial haplogroups define two phenotypes of osteoarthritis. Front Physiol 2012;3:129.

9 Mishmar D, Ruiz-Pesini E, Golik P, et al. Natural selection shaped regional mtDNA variation in humans. Proc Natl Acad Sci USA 2003:171-6.

10 Ruiz-Pesini E, Mishmar D, Brandon M, et al. Effects of purifying and adaptive selection on regional variation in human mtDNA. Science 2004;303:223-6.

11 Gómez-Durán A, Pacheu-Grau D, López-Gallardo E, et al. Unmasking the causes of multifactorial disorders: OXPHOS differences between mitochondrial haplogroups. Hum Mol Genet 2010;19:3343-53.

12 Kenney MC, Chwa M, Atilano SR, et al. Inherited mitochondrial DNA variants can affect complement, inflammation and apoptosis pathways: insights into mitochondrial-nuclear interactions. Hum Mol Genet 2014:23:3537-51.

13 Hudson G, Nalls M, Evans JR, et al. Two-stage association study and meta-analysis of mitochondrial DNA variants in Parkinson disease. Neurology 2013;80:2042-8.

14 Achilli A, Olivieri A, Pala M, et al. Mitochondrial DNA backgrounds might modulate diabetes complications rather than T2DM as a whole. PLOS ONE 2011;6:e21029.
15 Niemi AK, Hervonen A, Hurme $M$, et al. Mitochondrial DNA polymorphisms associated with longevity in a Finnish population. Hum Genet 2003:112:29-33.

16 Rego-Perez I, Fernandez-Moreno M, Fernandez-Lopez C, et al. Mitochondrial DNA haplogroups: role in the prevalence and severity of knee osteoarthritis. Arthritis Rheum 2008;58:2387-96.

17 Rego I, Fernandez-Moreno M, Fernandez-Lopez C, et al. The role of European mtDNA haplogroups in the prevalence of hip osteoarthritis in Galicia (Northern Spain). Ann Rheum Dis 2010;69:210-13.

18 Fang $H$, Liu $X$, Shen L, et al. Role of mtDNA haplogroups in the prevalence of knee osteoarthritis in a southern Chinese population. Int J Mol Sci 2014;15:2646-59.

19 Soto-Hermida A, Fernández-Moreno M, Oreiro N, et al. mtDNA haplogroups and osteoarthritis in different geographic populations. Mitochondrion 2014;15:18-23.

20 Hudson G, Panoutsopoulou K, Wilson I, et al. No evidence of an association between mitochondrial DNA variants and osteoarthritis in 7393 cases and 5122 controls. Ann Rheum Dis 2013;72:136-9.

21 Soto-Hermida A, Fernandez-Moreno M, Oreiro N, et al. Mitochondrial DNA (mtDNA) haplogroups influence the progression of knee osteoarthritis. Data from the Osteoarthritis Initiative (OAI). PLOS ONE 2014;9:e112735.

22 Soto-Hermida A, Fernandez-Moreno M, Pertega-Diaz S, et al. Mitochondrial DNA haplogroups modulate the radiographic progression of Spanish patients with osteoarthritis. Rheumatol Int 2015:35:337-44.

23 Felson DT, Niu J, Guermazi A, et al. Defining radiographic incidence and progression of knee osteoarthritis: suggested modifications of the Kellgren and Lawrence scale. Ann Rheum Dis 2011;70:1884-6.

24 Thorstensson CA, Andersson ML, Jönsson $\mathrm{H}$, et al. Natural course of knee osteoarthritis in middle-aged subjects with knee pain: 12-year follow-up using clinical and radiographic criteria. Ann Rheum Dis 2009;68:1890-3.

25 Van Spil WE, Welsing PM, Kloppenburg M, et al. Cross-sectional and predictive associations between plasma adipokines and radiographic signs of early-stage knee osteoarthritis: data from CHECK. Osteoarthritis Cartilage 2012:20:1278-85

26 DerSimonian R, Laird N. Meta-analysis in clinical trials. Control Clin Trials 1986;7:177-88

27 Chomyn A. Platelet-mediated transformation of human mitochondrial DNA-less cells. Meth Enzymol 1996;264:334-9.

28 Shen JM, Feng L, Feng C. Role of mtDNA haplogroups in the prevalence of osteoarthritis in different geographic populations: a meta-analysis. PLOS ONE 2014;9:e108896

29 Rego-Perez I, Fernandez-Moreno M, Soto-Hermida A, et al. Mitochondrial genetics and osteoarthritis. Front Biosci (Schol Ed) 2013;5:360-8.

30 Fernandez-Moreno M, Soto-Hermida A, Pertega S, et al. Mitochondrial DNA (mtDNA) haplogroups and serum levels of anti-oxidant enzymes in patients with osteoarthritis. Bmc Musculoskelet Disord 2011:12:264.

31 Ospelt C, Gay S. Somatic mutations in mitochondria: the chicken or the egg? Arthritis Res Ther 2005:7:179-80.

32 Wesseling J, Bierma-Zeinstra SM, Kloppenburg $M$, et al. Worsening of pain and function over 5 years in individuals with 'early' $O A$ is related to structural damage: data from the Osteoarthritis Initiative and CHECK (Cohort Hip \& Cohort Knee) study. Ann Rheum Dis 2015:74:347-53.

33 Wesseling J, Dekker J, van den Berg WB, et al. CHECK (Cohort Hip and Cohort Knee): similarities and differences with the Osteoarthritis Initiative. Ann Rheum Dis 2009:68:1413-19.

34 Rego-Pérez I, Fernández-Moreno $M$, Deberg $M$, et al. Mitochondrial DNA haplogroups modulate the serum levels of biomarkers in patients with osteoarthritis. Ann Rheum Dis 2010;69:910-17.

35 Rego-Pérez I, Fernández-Moreno M, Deberg M, et al. Mitochondrial DNA haplogroups and serum levels of proteolytic enzymes in patients with osteoarthritis. Ann Rheum Dis 2011:70:646-52.

36 Fernandez-Moreno M, Tamayo M, Soto-Hermida A, et al. mtDNA haplogroup J modulates telomere length and nitric oxide production. Bmc Musculoskelet Disord 2011;12:283.

37 Hannan MT, Felson DT, Pincus T. Analysis of the discordance between radiographic changes and knee pain in osteoarthritis of the knee. J Rheumatol 2000;27:1513-17.

38 Zeggini E, Panoutsopoulou K, Southam L, et al. Identification of new susceptibility loci for osteoarthritis (arCOGEN): a genome-wide association study. Lancet 2012:380:815-23.

39 Pierron D, Chang I, Arachiche A, et al. Mutation rate switch inside Eurasian mitochondrial haplogroups: impact of selection and consequences for dating settlement in Europe. PLOS ONE 2011;6:e21543.

40 Wallace DC, Brown MD, Lott MT. Mitochondrial DNA variation in human evolution and disease. Gene 1999:238:211-30.

41 Martínez-Redondo D, Marcuello A, Casajús JA, et al. Human mitochondrial haplogroup $\mathrm{H}$ : the highest V02max consumer-is it a paradox? Mitochondrion 2010;10:102-7

42 Liu-Bryan R. Inflammation and intracellular metabolism: new targets in $\mathrm{OA}$ Osteoarthr Cartil 2015;23:1835-42. 
43 Kenney MC, Chwa M, Atilano SR, et al. Molecular and bioenergetic differences between cells with African versus European inherited mitochondrial DNA haplogroups: Implications for population susceptibility to diseases. Biochim Biophys Acta 2014;1842:208-19.

44 Reed KN, Wilson G, Pearsall A, et al. The role of mitochondrial reactive oxygen species in cartilage matrix destruction. Mol Cell Biochem 2014;397:195-201.

45 van Gisbergen MW, Voets AM, Starmans MH, et al. How do changes in the mtDNA and mitochondrial dysfunction influence cancer and cancer therapy? Challenges, opportunities and models. Mutat Res Rev Mutat Res 2015;764:16-30.

46 Weng SW, Kuo HM, Chuang JH, et al. Study of insulin resistance in cybrid cells harboring diabetes-susceptible and diabetes-protective mitochondrial haplogroups. Mitochondrion 2013;13:888-97.
47 Hwang HS, Kim HA. Chondrocyte apoptosis in the pathogenesis of osteoarthritis. Int J Mol Sci 2015;16:26035-54.

48 Henrotin YE, Bruckner P, Pujol JP. The role of reactive oxygen species in homeostasis and degradation of cartilage. Osteoarthritis Cartilage 2003:11:747-55.

49 Roach HI. The complex pathology of osteoarthritis: even mitochondria are involved. Arthritis Rheum 2008;58:2217-18.

50 López-Armada MJ, Caramés B, Martín MA, et al. Mitochondrial activity is modulated by TNFalpha and IL-1beta in normal human chondrocyte cells. Osteoarthritis Cartilage 2006;14:1011-22.

51 Latorre-Pellicer A, Moreno-Loshuertos R, Lechuga-Vieco AV, et al. Mitochondrial and nuclear DNA matching shapes metabolism and healthy ageing. Nature 2016:535:561-5. 


\section{Correction: Mitochondrial DNA haplogroups influence the risk of incident knee osteoarthritis in OAI and CHECK cohorts. A meta-analysis and functional study}

Fernández-Moreno M, Soto-Hermida A, Vázquez-Mosquera M, et al. Mitochondrial DNA haplogroups influence the risk of incident knee osteoarthritis in OAI and CHECK cohorts. A meta-analysis and functional study. Ann of Rheum Dis 2017;76:1114-22. doi: 10.1136/annr heumdis-2016-210131

The second corresponding author was not listed.

The corresponding details are:

Francisco J Blanco MD PhD

Rheumatology Service

Clinical Research Unit

INIBIC-Complejo Hospitalario Universitario A Coruña

15006-A Coruña, Spain

flblagar@sergas.es

\section{(2) \\ OPEN ACCESS}

Open access This is an open access article distributed in accordance with the Creative Commons Attribution Non Commercial (CC BY-NC 4.0) license, which permits others to distribute, remix, adapt, build upon this work noncommercially, and license their derivative works on different terms, provided the original work is properly cited, appropriate credit is given, any changes made indicated, and the use is non-commercial. See: http://creativecommons.org/licenses/by-nc/ 4.0\%.

(C) Author(s) (or their employer(s)) 2019. Re-use permitted under CC BY-NC. No commercial re-use. See rights and permissions. Published by BMJ.

Ann Rheum Dis 2019;78:e143. doi:10.1136/annrheumdis-2016-210131corr1

(A) Check for updates 